\title{
A NOTE ON THE MINIMUM CONDITION
}

\author{
AVINOAM MANN
}

\begin{abstract}
We characterize the minimum condition on subgroups in terms of generators.
\end{abstract}

It is well known that the maximum condition on subgroups of a group $G$ is equivalent to each subgroup of $G$ being finitely generated (for simplicity we discuss only groups, but the discussion applies equally well to rings, modules, etc.). For the minimum condition, however, no characterization in terms of generators is usually given. The purpose of this note is to put on record the existence of such a characterization.

Definition. Let $S$ be an infinite subset of a group $G$. An element $s \in S$ is essential in $S$ if, given any infinite subset $T \subseteq S$, there exists an infinite subset $U \subseteq T$ such that the group $\langle U, s\rangle$ generated by $U$ and $s$ is different from the group $\langle U\rangle$ generated by $U$ alone.

THEOREM. A group $G$ satisfies the minimum condition on subgroups if and only if in any infinite subset of $G$ there are only finitely many essential elements.

Proof. Let the infinite subset $S$ contain infinitely many essential elements, and let $T$ be the set of these elements. Let $t_{1} \in T$. Then there exists an infinite subset $U_{1}$ of $T$ such that $\left\langle t_{1}, U_{1}\right\rangle=H_{1} \neq H_{2}=\left\langle U_{1}\right\rangle$. Let $t_{2} \in U_{1}$. Then there exists an infinite subset $U_{2}$ of $U_{1}$ such that $\left\langle t_{2}, U_{2}\right\rangle \neq\left\langle U_{2}\right\rangle=H_{3}$, so certainly $\mathrm{H}_{3} \neq \mathrm{H}_{2}$. Proceeding in the same way, we obviously get an infinite decreasing chain $H_{1} \supset$ $\mathrm{H}_{2} \supset \mathrm{H}_{3} \supset \cdots$.

Conversely, let $H_{1} \supset H_{2} \supset \cdots$ be an infinite properly decreasing chain of subgroups in $G$. Let each $i$ choose an element $s_{i} \in H_{i}-H_{i+1}$, and let $S=\left\{s_{i}\right\}$. Denote $S_{j}=\left\{s_{i} \mid i>j\right\}$. Then, given any infinite subset $T$ of $S, T \cap S_{j}$ is also infinite and $\left\langle T \cap S_{j}\right\rangle \neq\left\langle T \cap S_{j}, s_{j}\right\rangle$, because $T \cap S_{j} \subseteq H_{j+1}$. Thus all elements of $S$ are essential.

Note that the above proof shows that in the definition of essential elements we could take $T$ and $U$ to be cofinite in $S$.

The notion of essential elements could possibly be used to define other classes of groups, e.g., the condition that no infinite subset of $G$ contains essential elements if equivalent to all proper subgroups of $G$ by being finite.

INSTITUTE OF MATHEMATICS, HEBREW UNIVERSTTY, JERUSALEM, ISRARL

Received by the editors May 2, 1979.

AMS (MOS) subject classifications (1970). Primary 20E15.

(c) 1980 American Mathematical Society 0002-9939/80/0000-0537/\$01.25 